\title{
LUCIEN CUÉNOT, RICHARD GOLDSCHMIDT Y MIQUEL CRUSAFONT PAIRÓ
}

\author{
Adrià Casinos \\ Universidad de Barcelona. Departamento de Biología Animal \\ acasinos@ub.edu
}

Recibido: 17 junio 2014; Aceptado: 10 diciembre 2014.

Cómo citar este artículo/Citation: Casinos, Adrià (2016), "Lucien Cuénot, Richard Goldschmidt y Miquel Crusafont Pairó", Asclepio 68 (1): p126. doi: http://dx.doi.org/10.3989/asclepio.2016.05

RESUMEN: Se analiza un texto inédito de Miquel Crusafont Pairó, correspondiente a comunicación que llevó a cabo en la Fundació Bosch i Cardellach, sobre la necrológica de Lucien Cuénot publicada por Richard Goldschmidt. La comunicación es un fuerte ataque a Goldschmidt por sus críticas a la deriva teleológica de Cuénot en los últimos años de su vida.

PALABRAS CLAVE: Crusafont Pairó; Goldschmidt; Cuénot; Teilhard de Chardin; Finalismo.

\section{LUCIEN CUÉNOT, RICHARD GOLDSCHMIDT AND MIQUEL CRUSAFONT PAIRÓ}

ABSTRACT: An unpublished text, corresponding to a communication done by Crusafont Pairó is analysed. The communication is strongly critical with Cuénot's necrology published previously by Goldschmidt, where he regrets the teleological ideas on evolution that Cuénot maintained in the last years of his life.

KEY WORDS: Crusafont Pairó; Goldschmidt; Cuénot; Teilhard de Chardin; Finalism.

Copyright: () 2016 CSIC. Este es un artículo de acceso abierto distribuido bajo los términos de la licencia Creative Commons Attribution (CC BY) España 3.0. 


\section{INTRODUCCIÓN}

Lucien Cuénot (1866-1951), profesor de Zoología en la Universidad de Nancy, ha sido considerado como uno de los primeros mendelianos franceses. En efecto, sus investigaciones demostraron la herencia mendeliana de la pigmentación en los ratones (Cuénot, 1902-1906). Su actividad en ese ámbito quedó interrumpida por la Primera Guerra Mundial, que le obligó a evacuar su laboratorio de Nancy, con la consiguiente pérdida de las cepas con las que había llevado a cabo los experimentos (Gayon y Burian, 2000, p. 1101). Pero Cuénot fue también autor de una considerable obra sobre evolución, en especial sobre el problema de la adaptación, reflejada en diversos artículos (por ejemplo, Cuénot, 1901, 1909, 1914) y libros (Cuénot, $1921,1925,1941,1951)$. Por otro lado, fue el creador del concepto y del término «preadaptación» (Cuénot, 1914), tal y como es remarcado por diversos autores (Mayr, 1976, p. 100; Gayon, 1995, p. 335 y siguientes; Devillers y Guy, 1996, p. 3528).

Cuénot murió el 7 de enero de 1951. Ese mismo año Richard Goldschmidt ${ }^{1}$ publicó dos notas en referencia al citado zoólogo (Goldschmidt, 1951a, b), una necrológica, que vio la luz en el mes de marzo, y una recensión de la obra póstuma de Cuénot, L'Évolution Biologique. Les Faits. Les Incertitudes, publicada en setiembre.

El 28 de abril de 1951, el paleontólogo Miquel Crusafont Pairó leyó una comunicación en el pleno de la Fundació Bosch i Cardellach ${ }^{2}$ de Sabadell. Parece ser que dicha comunicación se ha mantenido inédita, si bien el manuscrito se encuentra depositado en la Biblioteca Miquel Carreras, de la misma ciudad de Sabadell.

El objeto del presente trabajo es describir y analizar el mencionado manuscrito de Crusafont Pairó, en el contexto de sus ideas finalistas, tradicionalmente vinculadas al teilhardismo, y de sus relaciones con otros científicos, no afines a su planteamiento ideológico. Ahora bien, dado que está basado en la necrológica de Cuénot hecha por Goldschmidt, el análisis también de ésta es indispensable.

\section{NECROLÓGICA DE LUCIEN CUÉNOT POR RICHARD GOLDSCHMIDT}

La nota necrológica (Goldschmidt, 1951a) comienza, con una referencia a los orígenes más bien humildes de Cuénot, sus estudios en la Sorbona, su especialización en zoología, a pesar de una temprana inclinación hacia la paleontología. A despecho de las oportunidades que habría tenido para trasladarse a París, desde 1890 desarrolló toda su carrera académica en la Universidad de Nancy, de la que sería emérito a partir de 1937. Por supuesto que Goldschmidt destaca la gran aportación de Cuénot a la genética mendeliana, en un estadio muy temprano de dicha rama de la biología, que supuso sus estudios sobre el control genético de la coloración de los ratones. Dichos estudios le valieron una fuerte polémica con T. H. Morgan ${ }^{3}$, quien dudaba de los resultados de Cuénot. Su último trabajo sobre el tema lo publicaría en 1911. De la citada investigación sobre la coloración pasó a interesarse por el factor hereditario del cáncer, también en los ratones. Los acontecimientos bélicos de 1914 supusieron la interrupción definitiva de esa línea, como se ha apuntado más arriba.

Goldschmidt rescata también otras aportaciones del zoólogo francés, como sus numerosos estudios sobre los invertebrados, pero sobre todo la introducción y desarrollo del concepto de "preadaptación», que es de una gran vigencia, a pesar de ciertos recientes olvidos, cuando se contrasta con el concepto de «exaptación» de Gould y Vrba (1982) ${ }^{4}$.

Goldschmidt dedica las últimas líneas de la necrológica a destacar la deriva filosófica de Cuénot en sus últimos años hacia el finalismo teleológico (Tétry, 1996, p. 742) cómo justificación de la complejidad de las «invenciones» de la naturaleza. El genético de Berkeley se lamenta de la deriva de Cuénot hacia la advocación de poderes místicos.

A careful study of these («tools» of animals; nota del autor) convinced him in later years that their origin cannot be understood without a teleological finalistic principle. It is remarkable that a great zoologist, with unusual command of all aspects of the facts,.. felt himself constrained in the end to become a defeatist by advocating mystical powers (Goldschmidt, 1951a, p. 310).

Pero al mismo tiempo lleva el agua a su molino, en su condición de crítico de la teoría de la síntesis (véase Gould, 1981, para una análisis en profundidad), aduciendo que la actitud de Cuénot era el resultado de apercibirse de las limitaciones del neodarwinismo más allá de los confines de la microevolución.

It will be good to realize that his attitude was the result of a clear realization that the Neo-Darwinism doctrine does not lead beyond the confines of microevolution and that the best rebutts (se respeta la ortografía original; nota del autor) of mysticism in this field will be the elaboration of new ideas that will bridge the gap left by Neo-Darwinists without recourse to defeatist philosophies (Goldschmidt, 1951a, p. 310).

A remarcar el uso del término «doctrine» en lugar de «theory», que sería lo esperable. 
Debe añadirse que Goldschmidt era un profundo conocedor de la obra de Cuénot. Ya en su obra Einführung in die Vererbungswissenschaft (1928, p. 233, 360) cita profusamente diversos trabajos del zoólogo francés sobre la herencia mendeliana de la pigmentación de los ratones. También lo cita diversas veces en su más conocido y polémico libro, The material basis of evolution, ya desde la introducción, en la que menciona dos libros del zoólogo de Nancy, La genèse des espèces animales (1911) y L'Adaptation (1925), entre los textos de teoría evolutiva importantes publicados previamente a su obra (Goldschmidt,1982[1940], p. 4).

En lo que respecta a la recensión de L'Évolution Biologique. Les Faits. Les Incertitudes (Goldschmidt, 1951b), se trata de un texto muy breve, de apenas 50 líneas, en formato de dos columnas, dedicadas en su mayor parte a citar las materias que cubre el libro. Solo en las últimas líneas el autor resalta la confesión cautelosa de Cuénot sobre su insatisfacción con las ideas evolucionistas modernas, resaltando que el zoólogo francés remarca la posibilidad de la herencia de caracteres adquiridos. También menciona Goldschmidt la propuesta adicional de Cuénot, de una filosofía finalista para entender la evolución.

...the author, although fully aware of the genetical study of evolution and its results, confesses rather cautiously, reiterating that most evolutionists will not follow him, that he is not satisfied with modern evolutionary ideas. He still thinks that a modified kind of doctrine of inheritance of acquired characters of the type proposed by Baldwin and Schmalhausen will be needed, and he feels the additional need, expressed in a noble spirit of self-criticism for a finalistic philosophy in order to understand evolution (Goldschmidt, 1951b, p. 310) $)^{5}$.

\section{LA COMUNICACIÓN DE MIQUEL CRUSAFONT PAIRÓ}

El manuscrito, comienza repitiendo los datos biográficos aportados por Goldschmidt, en su necrológica. El eje principal de la argumentación de Crusafont es la crítica a la reprobación que hacia aquel a las posiciones finalistas de Cuénot. De entrada, y de una manera un tanto forzada, sino casi maniquea, Crusafont confronta el pensamiento de Cuénot:

Como buen representante de la escuela francesa, Cuénot es un finalista, más o menos vagamente teísta (Crusafont, 1951a, p. 2).

al de Goldschmidt:

...el gran líder evolucionista norteamericano inspirado en las doctrinas tan materialistas de la escuela inglesa (Crusafont, 1951a, p. 3).
De hecho invierte la crítica de Goldschmidt a las posiciones filosóficas de Cuénot, que aquel consideraba "defeatist», en la medida que dichas posiciones le aparecen como insuficientes:

...considerando que Cuénot se ha refugiado en teorías filosóficas «defectistas» o "derrotistas» (traduzco la palabra «defeatist»). Ello apesar (sic) de la tibieza o ambigüedad de las ideas del gran biólogo francés... (Crusafont, 1951a, p. 2)

A continuación traduce una cita de Cuénot sobre la cuestión del antiazar:

Incluso no nos es prohibido detenernos en el agnosticismo, que admite sin más, la finalidad orgánica y que aplaza para el porvenir una explicación plausible del antiazar.

calificando sus palabras como:

...pobrísimo resto al fin y al cabo de un fermento de formación europea, ya dudosamente cristiana (Crusafont, 1951a, p. 3).

La crítica de Crusafont a la, a su vez, crítica de Goldschmidt la acaba estableciendo un símil que, en aquel momento histórico, era bastante vejatorio. Ni más ni menos que evoca indirectamente a Lysenko escribiendo:

El ataque de Goldschmidt contra la fortaleza de papel (siempre en el plano filosófico) de Cuénot, nos recuerda en otro grado lo que a su vez esgrimen los michurinistas soviéticos atacando con furibundez las teorías neodarwinistas inglesas y americana (Crusafont, 1951a, p. 3).

La comparación es tanto más ofensiva cuanto Goldschmidt se limita a llevar a cabo una crítica legítima, independientemente de que pudiera o no ser correcta.

La comunicación acaba con un párrafo confuso, que no resulta fácil analizar. Después de un alegato a favor de la obra divina y de su comprensión por parte del hombre de ciencia, advierte contra el peligro de que el científico traspase los límites de su disciplina para inmiscuirse en la filosofía:

...comprender por si (ortografía original; nota del autor) mismo cuanta maravilla nos ha sembrado para nuestro regalo espiritual, pero no debe pasar más allá de los setos que limitan su propio campo. Cuando no existen miras políticas o interesadas el científico debe tener las manos libres para trabajar científicamente, sin pretender entrar con derechos infundados, en el campo filosófico. Siempre se ha demostrado que el hombre de laboratorio, el experimentalista, resulta ser generalmente un malhadado científico (Crusafont, 1951a, p. 3).

(El subrayado es del propio Crusafont). 
Sorprende estas afirmaciones en contra de mezclar ciencia y filosofía, en el que sería con el tiempo uno de los máximos adalides de la difusión de la obra filosófica de Teilhard de Chardin.

Finalmente reprueba a Goldschmidt una vez más:

No resulta, para una mente equilibrada y creyente, un hecho verdaderamente aciago este de la diatriba de las doctrinas defectistas con que Goldschmidt inquieta en su tumba a nuestro gran biólogo que ya en vida tuvo la desgracia, filosóficamente hablando, de caminar sobre la cuerda floja del confusionismo ideológico? (Crusafont, 1951a, p. 3) ${ }^{6}$.

\section{DISCUSIÓN}

Diversos autores se han referido al conflicto que generaba en Crusafont Pairó su acendrado catolicismo, y los equilibrios que realizaba para hacerlo compatible con su aceptación de la evolución. Véase, por ejemplo, Acosta Rizo (2013) y Català Gorgues (2013) para una revisión reciente de la cuestión. No parece arriesgado asumir que la visión finalista del paleontólogo de Sabadell estaba causada por sus sentimientos religiosos, independientemente de su vinculación a las ideas de Teilhard de Chardin. Véase, por ejemplo, Crusafont Pairó (1948, 1951b). Crusafont Pairó (1951b), una revisión del tema de la evolución en España, acaba con un «Colofón" en el que hay frases que traslucen claramente el trasfondo ideológico de su evolucionismo, como:

En resumen: El Universo es finito, la materia creada es finita. Sólo Dios es infinito. (...) El hombre de ciencia puede sólo decir que es posible que el Producat terra sea ya una causa segunda derivada de la causa primera de la creación del Universo (Crusafont Pairó, 1951b, p. 174).

Cronológicamente hablando, la comunicación que aquí se analiza apunta a que la interpretación ortogenética de Crusafont es anterior a dichas vinculaciones con los núcleos theilardistas. Como señala Agustí (1993, p. 16) por los años en que está datada esta comunicación, Crusafont tenía un conocimiento muy precario de Teilhard. Previamente, su visión de la evolución estaba muy influenciada por un neolamarckismo vitalista próximo del de Cope o incluso Bergson. $Y$ la mayor influencia contemporánea que había recibido era la del paleontólogo italiano Alberto Carlo Blanc ${ }^{7}$. Sus primeras publicaciones sobre el pensamiento de Teilhard de Chardin datan de 1956 (Crusafont Pairó, 1956a,b). Cabe pues pensar, por cuestión cronológica, que en las teorías del jesuita francés Crusafont halló más bien una justificación o una cimentación a posteriori a su postura finalista. Por otro lado, la hipótesis de la vinculación unívoca entre creencias religiosas y finalismo falla por su base, cuando se piensa en otros científicos de su generación y de su entorno, quienes a pesar de su probado catolicismo, nunca fueron finalistas, sino opuestos al finalismo. El ejemplo quizá más conspicuo sería el de Ramon Margalef.

Podría incluso darse un ejemplo en cierto sentido opuesto, por lo que hace a la relación finalismo-catolicismo. Acosta Rizo (2013, p. 331) cita entre los amigos franceses de concepciones ortogenéticas a Jean-Pierre Lehman. Está adscripción de Lehman debe matizarse. Quien escribe considera que su posición era más bien la de admitir los argumentos ortogenéticos como inductores a reflexionar en torno a que el neodarwinismo no lo explicaba todo, posición que no era extraña entre los paleontólogos, sobre todo franceses, de su generación ${ }^{8}$. De la misma manera que consideraba interesantes los argumentos lamarckistas, aunque taxativamente afirmaba que él no creía en la herencia de los caracteres adquiridos (Lehman, 1962, p. 415). Por esa razón es algo osada la afirmación complementaria de Acosta Rizo (2013, p. 331) de no considerar a Lehman como darwinista. Lehman se permite incluso una boutade, teniendo en cuenta que él era conocido por ser una persona políticamente progresista9

En France en général la gauche est darwiniste, la droite lamarckienne (Lehman, 1962, p. 408)

Glick (1994, p. 50-51) cita la reacción que produjo en George Gaylord Simpson la afirmación de Lehman a propósito de los trabajos de Crusafont y Truyols sobre masterometría de carnívoros (Simpson, 1965). Para el paleontólogo francés los resultados eran una evidencia en contra de la teoría sintética; en ningún caso ponía el acento sobre la supuesta ortogénesis latente en las conclusiones, como hacían otros autores. Véase, como ejemplo, Pirlot (1976, pp. 364-365). Pero en el caso de que hubiera existido, la visión ortogenética de Jean-Pierre Lehman no tendría un trasfondo religioso, ya que dicho paleontólogo era un ateo convencido (Sigogneau-Russell, 2011). Es más, rechazaba totalmente por metafísica la idea de que la vida tendía y culminaba en la hominización (Lehman, 1962, p. 417).

Otro ejemplo es el mismo Goldschmidt (1940[1982], p. 395), quien, sin que parezca haber por en medio ninguna razón trascendente, en su obra más conocida asocia ortogénesis a macroevolución, a propósito de la preadaptación y citando a Schindewolf (1936).

A propósito de Goldschmidt en el texto de Crusafont destacan dos cosas, en cierta manera contradictorias. Por un lado su admiración y elogios hacia el genetista 
germano-estadounidense. Por el otro la agresividad de los ataques a su "materialismo" y al de sus pares anglosajones. Incluso Cuénot no sale tampoco indemne, ya que viene a decir que bastante pena tuvo aquel con profesar sus descarriadas ideas para que se le someta a críticas por sus vagas ideas de trascendentalidad.

Por lo que hace a la citada admiración hacia Richard Goldschmidt, vale la pena contextualizarla en lugar y tiempo. Goldschmidt no era precisamente un autor apreciado en la sección (luego facultad) de Biología de la Universidad de Barcelona, un lugar en el que el neodarwinismo llegó muy tempranamente, de la mano principalmente de Antoni Prevosti y Ramon Margalef, convirtiendo aquel centro en un baluarte. Basta consultar Margalef (1984, p. 52) con un cuestionamiento global de las hipótesis más o menos macroevolucionistas o, incluso, del cladismo. En efecto, en el método de Hennig veía Margalef una resurrección de las ideas de Daniele Rosa que, por supuesto, desaprobaba ${ }^{10}$. Curiosamente esa asociación entre Hennig y Rosa era defendida también por Léon Croizat, afín al cladismo (Croizat, 1978, p. 110), aunque con una valoración diferente, ya que veía plagio.

Por supuesto que las diferencias no se limitaban a la valoración de Goldschmidt. La visión evolucionista de Crusafont estaba a años luz de la dominante entre sus colegas biólogos de la Universidad de Barcelona. Entre ellos podía surgir de vez en cuando algún comentario en el sentido de reflejar saturación, respecto a la

\section{NOTAS}

1. Richard Goldschmidt (1878-1858), genetista de origen alemán, fue desde 1936 profesor en Berkeley. Se distinguió por sus puntos de vista heterodoxos, asumiendo que los fenómenos macroevolutivos respondían a mecanismos saltacionistas diferentes a los responsables de las variaciones intraespecíficas. La hipótesis del «monstruo prometedor» (hopeful monster) estaba basada en la creencia de que había genes especialmente importantes para el desarrollo, en los que una mutación podía originar cambios filogenéticos o especiativos. Véase Gould (1982).

2. La Fundació Bosch i Cardellach de Sabadell fue creada en 1942 por una serie de eruditos locales, entre los que figuraba el propio Miquel Crusafont, recuperando el nombre de Antoni Bosch i Cardellach (1758-1829), médico, historiador y archivero de la ciudad de Sabadell. En principio se constituyó como una institución dedicada a los estudios locales y comarcales, pero la comunicación de Crusafont que aquí se analiza iba por supuesto más allá de los fines propios. Cabe preguntarse hasta qué punto los asistentes eran conocedores de los personajes y cuestiones aludidas, y también si el tipo de reunión, que se puede imaginar en "petit comité», fue proclive al tono extemporáneo que Crusafont utilizó. insistencia de Crusafont en sus ideas "filosóficas»" Todo eso no era óbice para que Ramon Margalef, por ejemplo, sintiera un profundo respeto por su colega paleontólogo, no ya solo a nivel personal, sino también por su teilhardismo militante ${ }^{12}$.

Es en el marco de esa "coexistencia» entre neodarwinismo y finalismo, que incluye al propio Crusafont, quien no parece que fuera sectario en el círculo de colegas con los que mantenía una relación más o menos estrecha, círculo que incluía tanto a científicos ideológicamente afines a él (Joannes Hürzeler, Jean Piveteau) $)^{13}$, como otros que no lo eran (por ejemplo, los ya mencionados Georges G. Simpson y Jean-Pierre Lehman) ${ }^{14}$, que sorprende la argumentación y el tono que Crusafont utiliza en la comunicación objeto de este artículo. En efecto, ambos se podrían calificar de duros, incluso de hirientes e intolerantes. Cabe preguntarse si Crusafont hubiera utilizado tal tipo de estilo si en lugar tratarse de una comunicación oral, el texto hubiera ido destinado a su publicación. Entre otras cosas porque quizá el editor de la posible revista le hubiera pedido moderarse.

\section{AGRADECIMIENTOS}

A Jean-Pierre Gasc (Muséum, París) por sus comentarios; a Lluís Serra y Joan Real (Universidad de Barcelona) por sus indicaciones bibliográficas e información sobre la Fundación Bosch i Cardellach, respectivamente. Este artículo es una extensión del proyecto CGL2011-23919 del Ministerio de Economía y Competitividad.

Debe añadirse que la fundación que nos ocupa era conocida humorísticamente en la ciudad, por semejanza fonética, con el nombre de "Pocs i Garbellats" (literalmente, "pocos y tamizados"), quizá porque la veían como algo elitista.

3. Thomas Hunt Morgan (1866-1945), genetista estadounidense, fue premio Nobel de Fisiología y Medicina en 1933, por su contribución a la teoría cromosómica de la herencia. Dudó de los resultados de Cuénot porque cuestionaba que éste hubiera obtenido dominantes puros, ya que los recesivos latentes habían aparecido antes de lo usual. Véase Darden (1991), p. 101 y sig, y Goldschmidt (1951a).

4. Casinos, Adrià (en revisión), "From Cuénot's préadaptation to Gould's exaptation. A review of the concept(s)".Biological Journal of the Linnean Society.

5. James Mark Baldwin (1861-1934) fue un psicólogo americano que propuso que las prácticas culturales podían actuar sobre el genoma vía mecanismos epigenéticos. Véase Gayon (1996) p. 203. Ivan Ivanovich Schmalhausen (1884-1963), morfólogo soviético, es el autor del concepto de «selección estabilizadora», que implicaría la eliminación de las desviaciones respecto a la forma norma, con el remplazo subse- 
cuente de los factores externos por los factores internos de desarrollo. Carece de lógica considerar que Schmalhausen aceptaba la herencia de los caracteres adquiridos. Véase Berg (1996) p. 3832 y sig. y Wake (1986) p. IX.

6. Se ha trascrito este párrafo tal y cómo lo escribió Crusafont, es decir sin el signo de interrogación que debiera abrirlo. Cabe la posibilidad de que utilizara una máquina de escribir de teclado francés, dada la gran cantidad de tildes a mano en según qué vocales.

7. Alberto Carlo Blanc (1906-1960), paleontólogo italiano, fue autor de la teoría de la "cosmolisis», según la cual la diversidad biológica derivaría de un polimorfismo originario por un proceso de segregación de caracteres. Véase Blanc (194243) y Blanc y Blanc (2014).

8. Comunicación personal de Jean-Pierre Gasc, profesor emérito del Muséum de París.

9. Comunicación personal de Jean-Pierre Gasc, profesor emérito del Muséum de París.

10.Se trata de un comentario que Ramon Margalef hizo personalmente al autor en 1987, con motivo de un seminario dado por éste en la Facultad de Biología de la Universidad de Barcelona, sobre la lectura cladista del concepto de homología. Curiosamente, Crusafont, al menos en un primer momento, consideraba a Rosa "neodarwinista», aunque cabe pensar que no lo decía en el sentido actual, ya que data la teoría de la hologénesis de ese biólogo italiano en 1909. Véase Crusafont (1951b) p. 164. Sobre Rosa y su teoría, véase La Vergata (1996, p. 3729).

11.El autor fue testigo de que el rechazo hacia el teilhardismo de Crusafont era mayor entre los geólogos que entre los biólogos, y especialmente entre los estudiantes. La asignatura de Paleontología que impartía Crusafont era obligatoria para los estudiantes de geología y optativa para los de biología. Los primeros estaban especialmente quejosos de las continuas alusiones, por parte del profesor, a Teilhard de Chardin

\section{BIBLIOGRAFÍA}

Acosta Rizo, Carlos (2013), "Un pie en el cielo y otro en la ciencia. Las interacciones productivas de Miquel Crusafont", Dynamis, 33 (2), pp. 321-342.

Agustí, Jordi (1993), "El evolucionismo meridional de Miquel Crusafont”, Paleontologia i Evolució, 26-27, pp. 15-23.

Berg, Raïssa L. (1996), "Schmalhausen Ivan Ivanovič". En: Tort, Patrick (ed.), Dictionnaire du darwinisme et de l'évolution. Paris, Presses Universitaires de France, pp. 3825-3851.

Blanc, Alberto Carlo (1942-43), "Cosmolisi. Interpretazione genetico-storica delle entità e degli aggruppamenti biologici ed emologici", Rivista di Antropologia, 34, pp. 144-290.

Blanc, Gian Alberto; Blanc, Maria Cristina (2014), "Blanc, Alberto Carlo". En: Dizionario Biografico degli Italiani, 10. 1968. Treccani.it Enciclopedia Italiana, [en línea], [consultado el 09/012/2014]. o cuestiones afines, en unas clases que consideraban debían ser puramente empíricas.

12. El autor puede dar testimonio personal de que la participación de Miquel Crusafont Pairó (evocada por Acosta Rizo, 2013, p. 336) en las jornadas inaugurales del edificio de Biología en 1982, coincidiendo con el centenario de la muerte de Charles Darwin, se debió en gran parte a una iniciativa de Ramon Margalef, quien por supuesto era consciente de que una intervención de Crusafont giraría en torno a las ideas de Teilhard, como así fue (Crusafont, 1984). Por su parte Crusafont, ya enfermo y con dificultades para hablar, tenía cierta reticencia en participar. Aproximadamente un mes antes de dichas jornadas, en una conversación que el autor sostuvo con él en Sabadell, dijo, más o menos lo siguiente: "¿Qué quieren que explique? Ya he dicho todo lo que tenía de decir». El texto publicado (Crusafont, 1984) no da idea de la brevedad de la intervención, que además causó mucha tristeza, dada su deteriorado estado de salud.

13. Jean Piveteau fue un notable seguidor de Teilhard de Chardin y contribuyó a la divulgación y publicación de su obra (Piveteau, 1964, 1971). Por su parte Joannes Hürzeler bregó por conciliar fe y evolución, también desde su afinidad con las ideas de Teilhard. Calificaba la síntesis del jesuita de «grandiosa y genial tentativa» (Hürzeler, 1965, p. 117), si bien parecía más proclive que otros teilhardistas a admitir que la evolución podía ser o no al azar (Hürzeler, 1965, p. 142). Que finalmente las relaciones entre ese paleontólogo suizo y Crusafont se deterioraran, no fue en absoluto un problema ideológico, sino crematístico. Véase Galobart et al. (2011, p. 155).

14. El mutuo aprecio que se tenían Simpson y Crusafont, a pesar de sus diferentes puntos de vista sobre el tema religiónciencia, ha sido analizado por Acosta Rizo (2013), Català Gorgues (2013) y Glick (1994), como ya se ha indicado en el texto. En lo que se refiere a Lehman, al autor le consta personalmente la amistad que unía al paleontólogo francés con Crusafont.

Català Gorgues, Jesús I. (2013), “Miquel Crusafont, Georges Simpson y la internacionalización de los estudios de paleontología evolutiva en España", Dynamis, 33 (2), pp. 343-364.

Croizat, Léon (1978), “Hennig (1966) entre Rosa (1918) y Lovtrup (1977): Medio siglo de sistemática filogenética", Boletín de la Academia de Ciencias Físicas, Matemáticas y Naturales (Caracas), 38, pp. 59-147.

Crusafont Pairó, Miquel (1948), "Concepciones cosmovitalistas del evolucionismo", Publicaciones del Museo de Sabadell, fascículo IV.

Crusafont Pairó, Miquel (1951a), "Comentario a una nota necrológica sobre Lucien Cuénot", Fundació Bosch i Cardellach, Sabadell (manuscrito), pp. 1-3.

Crusafont Pairó, Miquel (1951b), “El tema de la evolución orgánica en España”, Estudios Geológicos, 7, pp. 159-175. 
Crusafont Pairó, Miquel (1956a), “El pensamiento científico del P. Teilhard de Chardin”, Estudios Geológicos, 31-32, pp. 344-375.

Crusafont Pairó, Miquel (1956b), “El fenómeno humano según los puntos de vista del P. Teilhard de Chardin, S.I.", Quaderns d'Arxiu de la Fundació Bosch i Cardellach, 23, 24 p.

Crusafont Pairó, Miquel (1984), “L'evolució i el fenomen humà segons Teilhard de Chardin". En: Sancho i Valls, Josep (coord.), Darwin a Barcelona. Barcelona, PPV, pp. 447-457.

Cuénot, Lucien (1901), "L'évolution des théories transformistes”, Révue générale des sciences pures et appliquées, 12, pp. 264-269.

Cuénot, Lucien 1909, "Le peuplement des places vides dans la nature et l'origine des adaptations", Révue générale des sciences pures et appliquées, 20, pp. 8-14.

Cuénot, Lucien (1914), "Théorie de la préadaptation”, Scientia, 16 , pp. 60-73.

Cuénot, Lucien (1921), La genèse des espèces animales, 2aㅡ edición. Paris, Félix Alcan.

Cuénot, Lucien (1925), L’Adaptation. Paris, G. Doin.

Cuénot, Lucien (1941), Invention et finalité en biologie. Paris, Flammarion.

Cuénot, Lucien (1951), L'Évolution Biologique. Les Faits. Les Incertitudes. Paris, Masson.

Darden, Lindley (1991), Theory Change in Science. Strategies from Mendelian Genetics. New York, Oxford University Press.

Devillers, Charles ; Guy, Yves (1996), "Pré-adaptation”. En: Tort, Patrick (ed.), Dictionnaire du darwinisme et de l'évolution. Paris, Presses Universitaires de France, pp. 3528.

Galobart, Angel; Acosta, Carlos A.; Vila, Bernat (2011), “Patrocinio y mecenazgo en paleontología de vertebrados: Crusafont, Hürzeler y Can Llobateres, diez años de una colaboración inacabada", Paleontologia i Evolució, Memòria especial 5, pp. 151-156.

Gayon, Jean (1995), “La notion de préadaptation dans l'oeuvre de Lucien Cuénot", Bulletin de la Société Zoologique de France, 120 , pp. 335-346.

Gayon, Jean (1996), "Baldwin James Mark". En: Tort, Patrick (ed.), Dictionnaire du darwinisme et de l'évolution. Paris, Presses Universitaires de France, pp. 202-204.

Gayon, Jean; Burian, Richard M. (2000), "France in the era of Mendelism (1900-1930)", Comptes Rendues de l'Académie des Sciences de Paris. Sciences de la vie, 323 (12), pp. 1097-1106.

Glick, Thomas F. (1994), “Miquel Crusafont i George Gaylord Simpson: interferències biogràfiques, confluències històriques", Arraona, 14, pp. 45-56.

Goldschmidt, Richard (1928), Einführung in die Vererbungswissenschaft, 5 a edición. Berlin, Julius Springer.
Goldschmidt, Richard (1982[1940), The material basis of evolution, New Haven and London, Yale University Press.

Goldschmidt, Richard (1951a), “L. Cuénot: 1866-1951”, Science, 113, pp. 309-310.

Goldschmidt, Richard (1951b), “L'Évolution Biologique: Les Faits, Les Incertitudes”, Science, 114, pp. 309-310.

Gould, Stephen Jay (1982), "The uses of heresy: an introduction to Richard's Goldschmidt The material basis of evolution". En: Goldschmidt, Richard, The material basis of evolution. New Haven and London, Yale University Press, pp. XIII-XLII.

Hürzeler, Joannes (1965), “El hecho de la evolución biológica. ¿Es posible el conocimiento exacto del decurso pasado de la evolución biológica?". En: Haag, Herbert; Haas, Adolf; Hürzeler, Joannes, Evolución y Biblia, traducción española. Barcelona, Herder, pp. 113-143.

La Vergata, Antonello (1996), “Rosa Daniele”. En: Tort, Patrick (ed.), Dictionnaire du darwinisme et de l'évolution. Paris, Presses Universitaires de France, pp. 3727-3730.

Lehman, Jean-Pierre (1962), "Paléontologie et théories modernes de l'évolution", L’Année Biologique, 1 (7-8), pp. 407-419.

Margalef, Ramon (1984), "El ritmo de la evolución en el marco cambiante de la biosfera". En: Sancho i Valls, Josep (coord.), Darwin a Barcelona. Barcelona, PPV, pp. 51-59, p.52.

Mayr, Ernst (1976), "The emergence of evolutionary novelties". En: Evolution and the diversity of life. Selected essays. Cambridge, Mass, The Belknap Press of Harvard University Press, pp. 88-113.

Pirlot, Paul (1976), Morfología Evolutiva de los Cordados, traducción española. Barcelona, Ediciones Omega.

Piveteau, Jean (1964) Le Père Teilhard de Chardin savant. Paris, Fayard.

Piveteau, Jean (1971), "Préface a Pierre Teilhard de Chardin". En: L'Oeuvre Scientifique, Textos reunidos y editados por $\mathrm{Ni}$ cole y Karl Schmitz-Moormann. Olten-Freiburg i.B., Walter Verlag, pp. XXI-XXIII.

Schindewolf, Otto H. (1936), Palaeontologie, Entwicklungslehre und Genetik. Berlin, Bornträger.

Sigogneau-Russell, Denise (2011), "Lehman, Jean-Pierre". En: Complete Dictionary of Scientific Biography. 2008. Encyclopedia.com, [en línea], disponible en: http://www.encyclopedia. com/doc/1G2-2830905217.html, [consultado el 13/09/2011].

Simpson, Georges Gaylord (1965), "A review of masterometry", Evolution, 19, pp. 249-255.

Tétry, André (1996), "Cuénot Lucien". En: Tort, Patrick (ed.), Dictionnaire du darwinisme et de l'évolution. Paris, Presses Universitaires de France, pp. 736-743.

Wake, David B. (1986). "Foreword, 1986". En: Schmalhausen, I.I., Factors of Evolution. The Theory of Stabilizing Selection. The University of Chicago Press, pp. V-XII. 